\title{
MINORANZE E SICUREZZA NAZIONALE IN MEDIO ORIENTE
}

\author{
di Alessandro Colombo
}

L'invasione del Kuwait e la guerra civile seguita alla sconfitta dell'Iraq hanno inferto un duro colpo alle semplificazioni più comuni della situazione mediorientale; quella, caratteristica delle fasi acute di tensione bipolare, che collocava le cause del conflitto fuori della regione, nella competizione Est/Ovest; quella, non meno parziale, centrata sul conflitto arabo-israeliano, che riportava all'interno del Medio Oriente le radici dell'instabilità ma non rinunciava a ricondurle tutte ad una sola; quella più recente, infine, del «nuovo ordine internazionale», che proprio dal «discorso» bipolare deduceva che, una volta venuto meno il conflitto tra le superpotenze, anche i conflitti regionali si sarebbero avviati a soluzione.

Il «nuovo ordine internazionale» nasce, in realtà, storicamente e concettualmente ambiguo (Freedman 1991). Storicamente, perché la fine della tensione Est/Ovest ha trascinato con sé la riemersione di conflitti vecchi e nuovi e il collasso di quegli elementi dell'ordine uscito dalla prima guerra mondiale che l'ordine uscito dalla seconda aveva per così dire integrato, come gli stati multinazionali succeduti agli Imperi. Concettualmente, poi, perché il diverso atteggiamento tenuto dalla «comunità internazionale» di fronte all'invasione irachena del Kuwait e al massacro degli insorti curdi e sciiti mostra già come scegliere una cosa significhi spesso in realtà sacrificarne un'altra, e come persino il rispetto di un principio astrattamente «pacifico» come quello dell'inviolabilità delle frontiere possa avere in concreto conseguenze paradossali.

La repressione dell' 'insurrezione in Iraq e l'esodo di milioni di profughi nei paesi confinanti hanno riportato in superficie una dimensione spesso trascurata nell'analisi internazionale: l'esistenza, in tutta la regione mediorientale, di diverse minoranze etniche e religiose, e l'incapacità da parte di tutti gli stati 
della regione di trovare una formula soddisfacente di convivenza.

Questo secondo aspetto sarà l'argomento delle prossime pagine. Che cosa rende così difficili i rapporti tra maggioranze $e$ minoranze? Quali sono le caratteristiche del problema in Medio Oriente? Più precisamente: per quale motivo la gestione dei rapporti con le minoranze prende forme quasi sempre conflittuali, che vanno dalla negazione dell'esistenza stessa dei gruppi minoritari, al rifiuto di concedere loro qualsiasi grado di autonomia, fino alla discriminazione sociale e giuridica e alla repressione vera e propria?

La tesi che proporremo è che questa precarietà derivi dallo stato di insicurezza nel quale versano i paesi della regione, che fa sì che l'esistenza delle minoranze venga trattata come una questione di «sicurezza nazionale» e le loro rivendicazioni come una minaccia all'esistenza dello stato. Il rapporto tra maggioranze e minoranze configura in Medio Oriente un tipico «dilemma della sicurezza» ${ }^{1}$ : anche se le rivendicazioni delle minoranze non hanno lo scopo di indebolire lo stato, lo stato non può essere sicuro che esse siano o restino pacifiche e quindi è indotto ad essere intollerante per difendersi. Se non che, a mano a mano che combatte le minoranze, lo stato rischia di indebolire la propria identità e di rafforzare la loro, precipitando in uno stato di insicurezza ancora peggiore.

Questa tesi ha due versanti, uno negativo e uno positivo. Il primo è il rigetto delle interpretazioni «polemiche» (nel senso anche etimologico del termine) che vedono nella presunta arretratezza della cultura arabo-islamica la causa di tutti i mali della regione, senza distinguere quello che gli attori fanno in quanto «arabi» e/o «islamici» da quello che fanno in quanto «attori» di un sistema competitivo (Allison 1971). A questa visione, che è ad un tempo «riduzionista» (Waltz 1979) - poiché colloca la causa belli nelle caratteristiche interne delle unità - e «idealistica» perché suggerisce una «conversione» alla democrazia, alla modernizzazione o all'Occidente, che eliminerebbe la guerra eliminando la bellicosità degli attori - si può rimproverare un eccesso di ottimismo e uno di pessimismo: di ottimismo, perché presuppone che basti cambiare le caratteristiche delle unità per ottenere la pace, senza considerare che buona parte di quello che fanno non dipende da come «sono» ma dalle condizioni in cui ope-

1 Sul concetto di «dilemma della sicurezza» cfr. le classiche esposizioni di Herz (1951) e Jervis (1978). 
rano; di pessimismo, paradossalmente, perché «convertire» qualcuno che non vuol essere convertito significa, con qualunque linguaggio lo si voglia esprimere, muovergli guerra.

Il versante positivo si riferisce invece, prima di tutto, alla scelta di un diverso «livello analitico» (Singer 1969), il sistema regionale, le cui «condizioni di costrizione» (Waltz 1979) agiscono sugli attori mediorientali nello stesso modo come agirebbero su qualunque altro attore nella stessa situazione. Utilizzando la nozione di «insicurezza» come criterio esplicativo osserveremo che gli stati della regione hanno diversi motivi per sentirsi insicuri, e che in condizioni di insicurezza quello che serve alle minoranze per affermare la propria identità e sopravvivere come gruppo viene automaticamente percepito dagli stati nei quali vivono come una minaccia diretta a loro. Lo scopo che ci prefiggiamo è dimostrare come, per capire perché l'esistenza dei gruppi minoritari sollevi tanta apprensione negli stati del Medio Oriente, non sia necessario risalire alle loro specificità culturali o religiose, ma basti sottolineare quelle caratteristiche del sistema mediorientale di fronte alle quali anche chi ha una cultura diversa tenderebbe ad agire nello stesso modo.

Per individuarle divideremo la nostra analisi in due parti. Nella prima ci soffermeremo sulla causa fondamentale dell'insicurezza, «l'artificialità dei confini», che allude alla mancanza di divisioni consolidate tra uno stato e l'altro e alla presenza di altre divisioni all'interno e al di sopra di quelle statuali. Per «artificialità dei confini» si intenderà, in altre parole, l'artificialità degli stati che delimitano, e cioè l'assenza (con la significativa eccezione dell'Egitto) di una identità nazionale che possa essere fatta valere all'interno e all'esterno contro tutte le altre.

Nella seconda parte, infine, vedremo quali altri elementi si innestano su quello principale. L'insicurezza degli attori verrà attribuita, oltre che alla difficoltà di definire la propria identità come distinta da quella degli altri, a quattro gruppi di ragioni «aggiuntive»: storiche, politico-strategiche, geo-politiche e, nel punto di confluenza delle altre, percettive.

\section{Alcune premesse}

Per gli scopi di questo saggio, per minoranza etnica o religiosa ${ }^{2}$ intenderemo molto genericamente qualunque collettività

${ }^{2}$ La definizione tiene conto dei lavori di Smith (1981); Brown (1989); Heraclides (1989); Riggs (1988); Ryan (1988). 
che si rappresenti e venga rappresentata dagli altri come distinta da quella maggioritaria entro cui vive, o almeno da quella che controlla l'apparato centrale dello stato. Il fondamento dell'identità è quello che è stato definito un confine «psicologico», o direttamente «etnico» (Heraclides 1989), tra interno ed esterno, membri e non membri, che può sorgere su terreni diversi quali la lingua, la religione, la storia, l'isolamento geografico o la razza, in diversa combinazione fra loro.

A questa definizione è necessario aggiungere alcune considerazioni.

1) Quando si parla di un problema delle minoranze non si deve dimenticare che dietro questa etichetta si confondono realtà spesso molto diverse (Clay 1989).

Innanzitutto varia radicalmente la relazione col potere, poiché accanto a situazioni nelle quali una maggioranza domina su una minoranza esistono situazioni nelle quali è una minoranza che domina su una maggioranza o su altre minoranze. Esempi di questo diverso rapporto sono la Siria, governata dalla minoranza alawita, e per certi versi anche l'Iraq, la cui classe dirigente è reclutata tra arabi di religione sunnita che rappresentano meno di un terzo della popolazione.

Un problema più complesso è dato dal fatto che il confine tra maggioranza e minoranza non è affatto fisso, anzi varia ogni volta che variano i riferimenti linguistici, religiosi o culturali in base ai quali lo stato e gli individui definiscono la propria identità. È la scelta tra i diversi criteri possibili di affiliazione, come la comunanza di lingua, di religione o di origine etnica, che determina «con chi» e «contro chi» stare, e quindi chi è «incluso» e chi è «escluso» dal gruppo. In questo senso, la divisione tra maggioranza e minoranza è una questione prospettica, se non il risultato di un conflitto «simbolico» su dove va posto il confine tra il «sé» e l'«altro». Non è un caso, per esempio, che proprio tra $\mathrm{i}$ cristiani d'Oriente siano emersi diversi tra i maggiori esponenti del nazionalismo arabo, tra $\mathrm{i}$ quali il principale teorico del partito nazionalista laico Baath, Michel Aflaq (Rodinson 1980), poiché è evidente l'interesse che essi hanno ad orientare l'affiliazione verso la comunanza linguistica e culturale, che li include nella maggioranza come arabi, a detrimento dell'identificazione religiosa che li esclude come cristiani (Cachia 1987).

Il problema delle minoranze, infine, varia considerevolmente anche per intensità, e il diverso grado di conflitto dipende, oltre che dalle caratteristiche degli stati, dalle caratteristiche dei 
gruppi minoritari, dalle loro dimensioni, dalle loro richieste e dai mezzi che impiegano (Heraclides 1989).

Un fattore critico è la sovrapposizione fra criteri diversi di affiliazione (Weinbaum 1987). Mentre la trasversalità delle identità etniche e religiose, come quella degli arabi di religione cristiana o dei berberi di religione islamica, tende a mitigare il conflitto lasciando aperti alcuni canali di comunicazione, la loro coincidenza irrigidisce la polarizzazione sociale. Esempi di questa spaccatura sono il conflitto tra curdi e persiani in Iran, che riflette oltre che una frattura etnica la frattura religiosa tra sunniti e sciiti, quello libanese tra i diversi gruppi islamici e i cristiani maroniti, che è stato aggravato dalla progressiva occidentalizzazione della comunità cristiana, e soprattutto quello tra arabi ed ebrei in Israele, che somma fratture di carattere culturale, etnico, religioso e linguistico.

Non meno importante è l'esistenza e il grado di contiguità territoriale (Riggs 1988; Francis 1976). Se i membri di una minoranza sono dispersi all'interno della popolazione e non hanno in mente alcun territorio definito - oppure, quando si tratta di immigranti, hanno in mente un territorio che è al di fuori del paese nel quale sono immigrati - il conflitto verte sui limiti dell'assimilazione o del rispetto delle identità culturali: in questi termini va vista la situazione delle minoranze religiose cristiane tollerate in quasi tutti i paesi della regione, o di quelle Bahai dichiarate fuorilegge in Marocco, Egitto e Siria dal 1960, in Iraq dal 1970 e in Iran, dove sono più numerose, dal 1979 (NES 1990/91). Il problema è più serio quando la minoranza è legata ad un determinato territorio, perché lo occupa o perché lo considera parte della propria identità. In questi casi, che sono stati definiti di «etnicità primaria» (Riggs 1988), il conflitto si sposta sui diversi gradi di autonomia territoriale, lungo un continuum che va dalla negazione pura e semplice fino all'indipendenza, e che può abbracciare una parte del territorio dello stato, come nel caso del Kurdistan in Iran, Iraq e Turchia, o persino l'intero territorio, come nelle formulazioni più radicali della questione palestinese.

2) Comunque si presenti, è necessario liberarsi dell'idea che quello delle minoranze sia un problema specificamente politico, o giuridico, o culturale. Dal momento che quasi sempre le componenti delle società multietniche sono diseguali nel numero, nel territorio e nelle capacità economiche, le divisioni etniche o religiose si traducono in una separazione sociale, mentre la se- 
parazione sociale rafforza il senso di appartenenza al gruppo etnico (Ryan 1988). Un esempio di questa interazione è il rapporto tra arabi ed ebrei in Israele, nel quale l'esistenza di obiettive diseguaglianze sociali, il modo come esse vengono percepite, e la separazione culturale, linguistica e religiosa della popolazione palestinese si alimentano reciprocamente. Fare notare che nella striscia di Gaza, dove è più forte il movimento islamico Hamas, circa 2.500 coloni ebrei posseggono il $28 \%$ della terra mentre i circa 950.000 arabi devono accontentarsi del resto, o che ad ogni palestinese spettano ogni anno 250 metri cubi di acqua contro i 27.600 che spettano ad ogni colono (Hottinger 1988), non significa tornare al rozzo determinismo che riconduceva ogni conflitto a fattori economici, ma piuttosto evitare di cadere nell'errore opposto di credere che i fattori economici non contino più niente.

3) È bene sottolineare, infine, che un problema delle minoranze esiste anche dove non compare, anzi spesso soprattutto dove non compare: l'evidenza è già, per certi versi, una forma rudimentale di riconoscimento.

Sono almeno tre gli accorgimenti utilizzati per occultare il problema, o presentarlo in termini parziali.

Il primo è quello che è stato detto «genocidio statistico», che consiste nella negazione dell'esistenza degli elementi diversi da quello maggioritario, secondo una utopia burocratica in base alla quale esiste solo quello che è autorizzato ad esistere. L'esempio più eclatante è l'assimilazione delle minoranze curde e armene nella Turchia kemalista (Peeters 1987), portata fino al divieto esplicito di nominarle e a disposizioni surreali come quella contenuta in una circolare del Ministero dell'Istruzione del 1983, che ordinava agli insegnanti di cancellare la parola «Armenia» da tutte le carte geografiche.

Un secondo accorgimento, di natura ideologica, è l'insistenza sull'unità degli arabi e/o dei musulmani, che confina nell'irrilevanza tutte le differenze al loro interno. Mentre il nazionalismo laico sminuisce i patriottismi locali, fondati su fattori specifici che dividono la «nazione araba», designandoli con nomi divenuti spregiativi come iqlimiyya, «regionalismo» (Rodinson 1980), l'enfasi del radicalismo religioso sull'unità della Casa dell'Islam rifiuta la nozione di identità linguistiche, etniche o nazionali, non perché ne ignori l'esistenza ma perché nega che questa conti qualcosa. «A volte» osserva l'Ayatollah Khomeini «il termine minoranza è usato per riferirsi a popoli come i cur- 
di, i turchi, i persiani, e così via. Ma questi popoli non dovrebbero essere chiamati minoranze perché questo termine dà per scontato che ci sia una differenza tra questi fratelli. Nell'Islam una simile differenza non trova posto. Non c'è differenza tra musulmani che parlano lingue diverse, per esempio gli arabi e i persiani. È molto probabile che questi problemi siano stati creati da coloro che non vogliono che i paesi islamici siano uniti» (Entessar 1989).

Un ultimo strumento, più ambiguo, è offerto dallo «schermo» della cittadinanza: i gruppi minoritari non compaiono in quanto non figurano come cittadini, come i palestinesi della diaspora o i numerosissimi lavoratori assunti a tempo determinato in Arabia Saudita e negli altri paesi del Golfo, ai quali i governi della regione non concedono il permesso di restare più a lungo proprio per prevenire la formazione di vere e proprie comunità etniche (Riggs 1988).

Il fatto che non sempre quello che è corretto sul piano giuridico aiuti a comprendere la realtà trova conferma nel caso dei palestinesi dei territori occupati, i quali pur senza essere formalmente cittadini israeliani (gli 800.000 della Cisgiordania hanno anzi passaporto giordano) hanno sconvolto il rapporto tra maggioranza e minoranza sia sul piano politico sia su quello strettamente numerico, portando la bilancia demografica di Israele da un rapporto ufficiale di 3.700 .000 ebrei contro 700.000 arabi ad uno complessivo di altrettanti ebrei contro 2.400.000 arabi, in rapido riequilibrio a causa del diverso tasso di crescita ${ }^{3}$. E solo alla luce di questa «gara demografica», oltre che per il timore di nuovi insediamenti, che è possibile comprendere perché i palestinesi abbiano percepito la nuova ondata di immigrazione ebraica dall'Unione Sovietica come una minaccia, o una sfida, o meglio ancora una conferma del proprio «stereotipo etnico»: quello di una maggioranza che, per ragioni che le sono estranee, diviene improvvisamente minoranza (Brinner 1987).

\section{Confini artificiali e insicurezza}

Invece di liquidare in partenza il problema attribuendo tutto quello che accade di spiacevole in Medio Oriente alle caratteri-

${ }^{3}$ Per stime più precise, cfr. NES (1990-1991); Brinner (1987, 14); Hottinger $(1988,308-309)$. 
stiche della cultura arabo-islamica, conviene sottolineare che, se la mancanza di una tradizione liberale aggrava certamente la situazione, altri elementi spiegano perché la presenza delle minoranze venga percepita come una questione di «sicurezza nazionale» (Clay 1989) e una minaccia all'esistenza dello stato.

Il principale mito da sfatare è che esista una intolleranza congenita nel mondo arabo o islamico, e che la situazione attuale non faccia che perpetuare questo atteggiamento. Anche se è rischioso generalizzare, si può affermare con una certa sicurezza che nel tradizionale dominio musulmano la posizione delle minoranze non fu affatto peggiore che nei paesi cristiani (Cahen 1969; Gronenbaum 1971), e che, pur restando sia in teoria che in pratica cittadini di secondo rango, cristiani ed ebrei non lo sono stati al punto da essere indotti a scegliere tra abbandonare il paese o lasciarsi assimilare (come i musulmani spagnoli dopo la Reconquista). I periodici scoppi di intolleran$z a$, associati spesso a periodi di debolezza politica o alla presenza di minacce esterne, come all'epoca dell'invasione mongola o nel corso della disgregazione dell'Impero turco, non devono far dimenticare anzi che proprio la mancanza di istanze centralizzatrici è alla base dell'attuale proliferazione di gruppi etnici e religiosi, e che se questo è segno di arretratezza politica essa è dovuta al fatto che, mentre in Europa lo stato moderno, soprattutto dopo la Rivoluzione francese, ha perseguito l'unificazione linguistica, giuridica e amministrativa del territorio cancellando ogni traccia di strutture intermedie ${ }^{4}$, l'Impero ottomano non ne ha impedito lo sviluppo (Weinbaum 1987) sia perché è stato sempre meno in grado di controllare le regioni periferiche sia perché, con il sistema dei millet, ha organizzato i principali gruppi religiosi in comunità relativamente autonome rette ciascuna dalle proprie leggi e da una propria organizzazione amministrativa (che comprendeva persino scuole, ospedali, un sistema assistenziale e uno giudiziario) (Gronenbaum 1971).

Un secondo elemento da tenere presente, per capire perché il problema delle minoranze assuma caratteri particolari, è l'esistenza nella regione di importanti segmenti di unità (Rodinson 1988). Il fattore unitario più evidente è la comunanza della lingua scritta, l'arabo classico, che oltre ad essere la sola lingua di cultura permette (grazie a qualche semplificazione e ad alcuni

4 Per la ricaduta di questa logica sul Medio Oriente, cfr. Entessar (1989, 86-87); Ryan (1988, 169 ss.). 
compromessi con i dialetti) la comprensione e la comunicazione reciproche. Ma notevoli fattori di unità si trovano anche nella cultura, nella mentalità collettiva e soprattutto nella memoria storica, nell'ambito della quale la storia comune degli arabi ha un rilievo centrale sia perché coincide con l'apogeo della loro storia sia perché, a differenza del passato preislamico, consente di conciliare la grandezza politica con il trionfo dell'Islam (Klein 1990).

Queste due caratteristiche, la molteplicità dei gruppi etnici e religiosi e la presenza di un retroterra unitario, fanno sì che gli stati mediorientali abbiano fondamenti molto diversi e molto più fragili rispetto a quelli europei. L'artificialità dei loro confini non deriva solo dal modo come sono stati fissati dalle potenze coloniali, ma dal fatto che, comunque fossero stati fissati, non sarebbe stato possibile innestare lo stato-nazione di tipo europeo su un'area ad un tempo unitaria e frammentata senza escludere qualcuno che non avrebbe dovuto essere escluso e senza includere qualcun altro che non avrebbe dovuto essere incluso.

Da una parte, non esiste tra i paesi arabi, che sono la grande maggioranza dei paesi della regione, una divisione linguistica, culturale e spesso neppure storica paragonabile a quella alla quale siamo abituati in Europa per distinguere ogni stato dagli altri. L'esterno, si può dire, non è diviso dall'interno, non perché il mondo arabo costituisca politicamente una unità, come sostengono i nazionalisti, ma perché le divisioni tracciate al suo interno hanno sempre oscillato tra forme estreme di frammentazione e sintesi più ampie di quelle degli stati attuali, senza mai pregiudicare il senso dell'unità dell'intera area o almeno di alcune sub-regioni relativamente definite (come quella persica attorno a Baghdad, o le due mediterranee attorno a Damasco e all'Egitto) (Cahen 1969; Gronenbaum 1971). In questo senso si può dire che quasi tutti i confini sono arbitrari, non perché il mondo arabo non dovrebbe averne ma perché non ci sono ragioni storiche, linguistiche o culturali perché essi cadano là dove cadono e non altrove.

Dall'altra parte, la pluralità dei gruppi etnici e religiosi e il fatto che le potenze coloniali hanno diviso la regione più in base ai propri interessi economici e di potenza che non alle fratture locali, fanno sì che tutti i paesi della regione (sia arabi sia non arabi) comprendano consistenti minoranze spesso legate a un territorio. Mentre possono non esserci, quindi, fratture sostanziali con l'esterno, fratture di natura linguistica, etnica e religiosa riemergono all'interno di ogni singolo stato. 
La logica che gli è propria è contraddetta su tutti e due i versanti. Sul primo, perché lo stato moderno rivendica la completa indipendenza politica dall'esterno, e quindi spezza tutti i legami per porsi come unico arbitro dei suoi destini. Sul secondo, perché esso ingaggia una lotta senza quartiere contro le autonomie politiche, giuridiche e linguistiche esistenti al suo interno, non ammettendo che altre divisioni indeboliscano l'unica divisione che consente, tra cittadini e non cittadini (Balladore Pallieri 1964). L'aspirazione dello stato moderno, come osservava Tocqueville all'indomani della Rivoluzione francese, è «formare una sola classe di cittadini» perché «questa superficie tutta eguale facilita l'esercizio del potere» (Tocqueville 1981). Quello che potrebbe essere diversamente viene livellato, cioè assimilato - come speravano e presumibilmente sperano ancora i teorici della modernizzazione, sia liberali che marxisti ${ }^{5}$ - o «reso eguale» per comodità burocratica ${ }^{6}$, come i curdi ribattezzati «turchi delle montagne» in nome del principio per il quale non possono vivere che cittadini «turchi» in uno stato «turco» (Entessar 1989; Ryan 1988).

Per inciso: per toglierci la solita tentazione di cercare residui premoderni in tutto quello che non ci piace del moderno, o della sua «dialettica», il caso ha voluto che negli stessi giorni in cui il governo e l'opinione pubblica francese premevano perché fosse riconosciuta in Iraq e in Turchia l'esistenza di un «popolo curdo» distinto da quello iracheno e turco, il Consiglio Costituzionale di Parigi dichiarasse illegittimo l'art. 1 del nuovo statuto della Corsica in quanto «la nozione di "popolo corso componente del popolo francese" in esso contenuta è contraria alla Costituzione, per la quale non esiste che il popolo francese, composto da tutti i cittadini francesi senza distinzione di origine, razza e religione».

Infine, questi consistenti gruppi etnici e religiosi «tagliano» quasi sempre il confine tra gli stati, contribuendo a indebolire anche in questo modo il loro limite. Così come l'esterno non è diviso dall'interno, l'interno non è diviso dall'esterno: la presen$z a$, nel territorio di ogni stato, di fratture linguistiche, etniche e

5 Sull'impatto della teoria della modernizzazione sullo studio delle minoranze etniche, cfr. Brown (1989); Ryan (1988).

6 Il rapporto tra eguaglianza dei cittadini e negazione delle identità è sottolineato in diversi studi sull'emancipazione illuminista e la «questione ebraica». Per una breve rassegna, cfr. Cremonesi (1985). 
religiose che mancano spesso tra uno stato e l'altro, ha effetti ancora più destabilizzanti in quanto, oltre a dividere quello che nelle divisioni di fonte statuale è unito, queste fratture uniscono quello che in esse è diviso, i territori e i cittadini di stati diversi.

Si possono distinguere tre forme di sovrapposizione, in un crescendo di pericolosità.

La prima si verifica quando una minoranza è minoranza in più stati (Weinbaum 1987), come i berberi in Africa settentrionale o $\mathrm{i}$ curdi in Iran, Iraq, Turchia e Siria. In questo caso all'interesse comune delle minoranze ad essere riconosciute come parti di un'unica minoranza, si contrappone l'interesse comune degli stati ad impedire che questo avvenga ${ }^{7}$.

La seconda, più grave, si verifica quando quella che in uno stato è la minoranza è la maggioranza in un altro, come gli arabi iraniani al confine con l'Iraq o gli sciiti libanesi appoggiati finanziariamente e militarmente dall'Iran. La presenza di popolazioni etnicamente simili o di correligionari al di là dei confini dello stato, soprattutto in paesi confinanti, crea un conflitto di fedeltà (al proprio stato «o» al proprio gruppo etnico o religioso) che rischia questa volta di sfociare in un conflitto tra stati.

La terza forma, la più pericolosa, ha luogo nei casi di reciprocità, e si verifica sia quando quello che è il gruppo maggioritario in un paese è il gruppo minoritario nell'altro, sia quando il gruppo che è minoranza in uno stato è maggioranza nella regione, mentre quello che è maggioranza nel primo è minoranza nella seconda (Ryan 1988). Il conflitto tra arabi e israeliani è l'esempio più emblematico di questa situazione.

L'artificialità dei confini crea una prima forma, e la più radicale, di insicurezza: quella sulla propria identità, sulla linea cioè che divide il sé e l'altro, l'interno e l'esterno, i cittadini e i non cittadini. In stati ancora incapaci nella maggior parte dei casi di suscitare forti sentimenti di lealtà, e persino di definire la propria identità come distinta da quella degli altri, le richieste delle minoranze vengono percepite come minacce proprio perché pongono una questione di identità che lo stato non ha ancora risolto.

Esiste, si può dire, un paradosso dell'artificialità, in virtù

7 Un esempio di questa comunanza di interessi, tanto più significativo in quanto avviene in una fase di notevole raffreddamento nelle relazioni tra i due paesi, è l'accordo di sicurezza siglato da Iran e Turchia nel settembre 1992 per la repressione dell'insurrezione curda. 
del quale quanto più i confini sono artificiali tanto più è necessario continuare a «fissarli» e a vietare che altre identità indeboliscano in un senso o nell'altro la divisione. È proprio quando non esiste una frattura evidente tra l'interno e l'esterno, il sé e l'altro, che diviene importante «inventarne» una e difenderla da tutte le definizioni diverse.

La posizione dei paesi arabi è la più precaria. L'esistenza di un forte sentimento unitario basato sulla comunanza etnica o religiosa, la mancanza di una tradizione statuale (con l'eccezione, come si è detto, dell'Egitto), e la scarsa legittimità dei confini di impronta coloniale, hanno reso difficilissima nella maggior parte dei casi la formazione di una identità nazionale distinta da quella degli altri (Baram 1990). I tentativi di attingere al passato prearabo e preislamico - come la riscoperta dell'eredità faraoni$\mathrm{ca}$ in Egitto negli anni '20 e più recentemente, non a caso, durante il governo Sadat, o quella di Cartagine in Tunisia, o ancora più significativa la ripresa della tradizione mesopotamica in Iraq (con l'organizzazione per esempio di una cerimonia annuale a Mossul, proprio dove l'identità «nazionale» irachena è più debole) e in Siria - sono andati incontro ogni volta alle stesse difficoltà; da una parte, l'accento sul «nazionalismo territoriale» (Hourani 1962; Baram 1990) contraddice i sentimenti unitari del mondo arabo e l'ortodossia ideologica dei partiti nazionalisti come quello Baath iracheno e siriano; dall'altra fondare la propria identità nazionale sul passato preislamico significa entrare in conflitto con l'altra fonte tradizionale di legittimità, quella religiosa, che svaluta tutte le civiltà preislamiche come tali (Baram 1990; Klein 1990).

Il risultato è una continua oscillazione tra fattori unitari e fattori di differenziazione, e nell'ambito di questi tra elementi attinti alla storia prearaba ed elementi che conferiscono un ruolo particolare, e centrale, nell'ambito della storia araba (come il califfato omayyade in Siria e quello abbaside in Iraq). Il paradosso dell'identità degli stati arabi è che per affermare l'unità politica degli arabi e/o degli islamici essi devono negarsi come stati, mentre per affermarsi come stati devono negare l'unità politica degli arabi e/o degli islamici.

La loro identità debole è intollerante, prima di tutto, perché è debole. Bisogna essere molto ottimisti per sperare che uno stato ancora incapace di rispondere in modo univoco alla domanda (che compare esplicitamente in un discorso di dieci anni fa del principe ereditario giordano): «chi siamo, e a che cosa 
apparteniamo» (Baram 1990, 439) possa tollerare che qualcun altro, al suo interno, dia risposte diverse. Il dramma delle minoranze nei paesi arabi è che ogni affermazione della loro identità è percepita dagli stati nei quali vivono come una negazione della propria (Heraclides 1989).

La posizione di Israele, per quanto molto diversa, non è meno istruttiva. Anche se, per lo stato ebraico, non ci sono difficoltà a definire la propria identità come distinta da quella degli altri paesi della regione, il problema si ripresenta per la minoranza araba e per i rapporti che questa mantiene con l'esterno. Dalla data della sua fondazione, Israele ha cercato di «inventare» una identità arabo-israeliana (Brinner 1987) che non coincide naturalmente con la propria, ma serve a spezzare il legame tra gli arabi che vivono sul suo territorio e tutti gli altri. Lo stesso problema di identità che impone agli stati arabi di alzare una frattura tra il «sé» e l'«altro» impone ad Israele di alzarne una nell'unico punto dove essa manca (dove, si può dire, il confine è aperto), e cioè tra i «propri» arabi e gli «altri» arabi, la propria minoranza e lo spazio dove essa torna ad essere maggioranza.

In un progetto per l'istruzione presentato alla Knesset nel 1976 si legge che le scuole ebraiche devono sottolineare «l'unicità dell'ebreo, e il "legame" tra la popolazione di Israele nel suo stato e la popolazione ebraica nella diaspora», mentre quelle arabe devono promuovere «l'amore della patria comune a tutti $\mathrm{i}$ cittadini dello stato (...) mettendo in rilievo gli interessi comuni e promuovendo "l'unicità" degli arabi di Israele» (Brinner 1987, 19). In un caso è affermata una identità etnica, culturale e religiosa che passa sopra all'identità territoriale, nell'altro una identità territoriale che spezza ogni traccia di identità etnica. L'unicità divide gli arabi di Israele sia dalla maggioranza ebraica sia dal resto degli arabi. Il governo israeliano riconosce sia giuridicamente sia politicamente l'esistenza di una minoran$\mathrm{za}$ al suo interno, ma non può riconoscere che questa minoranza sia parte di qualcosa che continua anche fuori dei suoi confini. Quella che Israele può riconoscere è, per così dire, una identità diversa da quella che essa rivendica. Stretti dalla paradossale necessità di essere ad un tempo arabi e israeliani, gli arabo-israeliani si sono trovati nella posizione di non sentirsi più né una cosa né l'altra (Moragh 1989).

Qualcosa di simile può valere oggi per i territori occupati. Se, da un lato, la guerra del '67 ha messo in crisi l'unicità degli 
arabo-israeliani rimettendoli in contatto con gli altri arabi sottoposti all'occupazione israeliana (Brinner 1987), dall'altro lato l'insistenza del governo Shamir perché ai negoziati di pace fossero ammessi solo i rappresentanti dei territori occupati riflette la solita preoccupazione di separare gli arabi sottoposti al proprio controllo da tutti gli altri, anche se questa volta il limite è spostato in avanti.

\section{Le altre fonti dell'insicurezza}

Ma l'apprensione che gli stati mediorientali mostrano di fronte alle rivendicazioni delle minoranze è aggravata anche da altre ragioni.

Ragioni storiche. Le passate collusioni delle minoranze con le potenze europee, il ruolo di intermediazione che hanno avuto per tutto il XIX secolo nella penetrazione del capitale europeo in Oriente (Moutafakis 1986), e soprattutto l'uso politico che di esse è stato fatto per indebolire l'Impero ottomano prima e ridisegnare la regione poi contribuiscono ad aumentare la diffidenza nei loro confronti, tanto più che questa diffidenza, che per una sorta di circolo vizioso spinge chi ne è colpito a cercare davvero un appoggio esterno, tende sempre a giustificarsi a posteriori (Weinbaum 1987).

La parabola dei cristiani libanesi è emblematica (Hagopian 1989). Dopo essere vissuti per più di un millennio in uno stato di relativa autonomia, la progressiva occidentalizzazione seguita alla penetrazione economica e culturale francese li ha allontanati sempre di più dall'arabismo, mentre il timore di perdere la propria identità ed essere assorbiti dal mondo arabo li ha legati sempre di più all'Occidente. Le patetiche riunioni di governo che il generale Aoun convocava ancora tra le mura dell'ambasciata francese nei giorni della sconfitta, mentre fuori il Libano era annesso di fatto alla Siria di Assad, sono l'immagine quasi letteraria di questo processo.

L'esperienza negativa degli ultimi due secoli influisce in diversi modi sulla condizione delle minoranze. Innanzitutto perché Francia e Gran Bretagna hanno seguito nella gestione dei loro rispettivi mandati una abile politica di contrapposizione di parte a parte della popolazione (Brown 1989; Cataluccio 1977), sostenendo alcune minoranze contro altre - come, in Libano, 
con l'appoggio incrociato della Francia ai maroniti e dell'Inghilterra ai drusi (che già l'Impero ottomano aveva utilizzato contro la maggioranza sunnita) - e soprattutto, secondo una consolidata pratica coloniale, concedendo il potere a minoranze anche esigue a scapito delle maggioranze - come fece la Francia in Siria con la piccola minoranza religiosa degli alawiti, e la Gran Bretagna in Iraq con gli arabi sunniti.

In secondo luogo perché la strumentalizzazione del problema da parte delle potenze europee, che col pretesto del rispetto delle minoranze hanno spesso cercato soltanto basi politiche ed economiche all'interno della regione, ha fatto sì che l'esistenza delle minoranze venisse via via considerata come una minaccia alla sicurezza e una potenziale «testa di ponte» per l'ingerenza straniera. La politica delle «capitolazioni» tra le potenze europee e l'Impero ottomano nel XVIII e nel XIX secolo, il legame che questa istituiva tra la penetrazione del capitale occidentale e il rispetto delle minoranze, la posizione di favore che queste ottennero nei rapporti economici con l'Europa (Moutafakis 1986; Gronenbaum 1972), e poi ancora l'uso spregiudicato che se ne è fatto per indebolire i paesi della regione od ottenere privilegi economici (come lo sfruttamento dei pozzi petroliferi in Iran a cavallo della prima guerra mondiale, per ottenere il quale gli inglesi appoggiarono le rivendicazioni della minoranza araba del Sud-Ovest iraniano contro il governo centrale) (Gronenbaum 1972), contribuiscono non poco a far sì che le minoranze vengano percepite non per quello che «sono» ma per la minaccia che «possono rappresentare». Da questo lato bisognerà vedere se, una volta esaurita la fase militare, l'intervento occidentale in Kurdistan non finirà proprio con l'aggravare questa percezione.

Ma l'esperienza che ha inciso di più su questo atteggiamento è il ruolo che le minoranze hanno giocato nelle politiche occidentali miranti alla frammentazione del mondo arabo. Come faceva osservare settant'anni fa il presidente della Commissione dei mandati alla XXV sessione, il diritto delle minoranze ad essere protette nelle loro caratteristiche etniche o religiose o linguistiche è stato spesso confuso col diritto a divenire «stato», col «diritto al separatismo» (Cataluccio 1977). La divisione già emblematica della Siria in quattro parti, nel 1920, che riconosceva una individualità statuale alla minoranza alawita, a quella ancora più esigua dei drusi, e creava un Libano politicamente cristiano con superficie raddoppiata rispetto ai confini del 
1914 , è stata superata 25 anni più tardi dall'esperienza destinata a diventare il cuore simbolico del problema delle minoranze in Medio Oriente, se non lo «stereotipo» attraverso il quale tutti gli stati della regione guardano più o meno strumentalmente alle proprie: la nascita dello stato di Israele, che se da una parte ha significato l'unica garanzia credibile della sopravvivenza politica della minoranza ebraica, dall'altra ha fissato un precedente, tanto più inquietante quanto più è debole l'identità dei paesi mediorientali, di come persino minoranze esigue come quella dei 650.000 ebrei del 1948 «minaccino» di pretendere, un giorno, la dignità di stato, disintegrando quelli che già esistono.

Ragioni politico-strategiche. Un secondo gruppo di ragioni è legato alla complessa interazione tra conflitti interni e conflitti esterni, e a quello che comporta sull'atteggiamento degli attori.

Il principale dato da tenere presente è l'altissima instabilità della regione e il frequente stato di conflitto tra i principali paesi. La regola per la quale «in ogni sorta di guerra i gruppi non sono tolleranti» (Simmel 1955) comporta che deviazioni che potrebbero essere tollerate, o negoziate, nei periodi «normali» non possano più esserlo in situazioni di crisi. Lo stato di tensione cronica della regione produce quello che potrebbe essere definito un caso di «determinismo dell'esterno sull'interno» (Bonanate 1986): una situazione cioè nella quale gli attori sono portati a regolare $\mathrm{i}$ propri rapporti politici interni in modo da poter sempre affrontare da posizioni di forza i rapporti politici esterni. Di fronte al continuo rischio di guerra le rivendicazioni delle minoranze sono accolte automaticamente come minacce, perché dividono lo stato e lo rendono più debole dei suoi potenziali nemici (Ryan 1988).

L'altra faccia del problema è l'«internazionalizzazione» dei conflitti interni (Weinbaum 1987). Il rapporto dei governi mediorientali con le minoranze è reso più difficile dal sostegno che queste ricevono, in forma politica, economica o persino militare, da altri governi. Che i gruppi minoritari continuino ad essere visti, come ai tempi della penetrazione europea, come strumenti almeno potenziali dell'ingerenza straniera, è una delle dimensioni fondamentali del problema. Quello che la rende più salda è l'impressionante serie di conferme che riceve; basti pensare al mosaico della guerra civile libanese, all'appoggio che la minoranza palestinese in Israele riceve dagli altri paesi arabi, al sostegno incrociato assicurato alla minoranza curda dall'Iran in 
Iraq e dall'Iraq in Iran (che ha avuto una significativa conferma proprio nel momento di massima insicurezza per entrambi, durante la prima guerra del Golfo) (Entessar 1989), ai rapporti degli sciiti iracheni con il governo iraniano, a cui l'Iraq potrebbe rispondere in futuro aumentando la sua influenza sulla minoranza araba in Iran. Il fatto che, in tutti questi casi, al disegno degli stati nemici di servirsi delle rispettive minoranze faccia da contraltare il tentativo delle minoranze di trascinare altri nel loro conflitto (Weinbaum 1987), non cambia la sostanza delle cose ma anzi peggiora la diffidenza nei loro confronti.

Ragioni geo-politiche. Almeno un accenno va fatto, inoltre, alla complicazione che insorge quando la minoranza è radicata ad un territorio che lo stato considera, per motivi economici o strategici, essenziale per la sua sicurezza. Che, in questi casi, le richieste di autonomia amministrativa vengano percepite come una minaccia all'esistenza dello stato, è un fraintendimento quasi scontato in un'area contrassegnata da confini deboli e dal «trauma» della trasformazione di altre minoranze in stati.

Esempi di questa sovrapposizione tra questione delle minoranze e questioni di «sicurezza nazionale» sono la situazione dei territori occupati in Israele (Cohen 1986), quella del Sud-Ovest iraniano attorno a Bassora e quella molto simile del Kurdistan turco o della zona di Mossul e Kirkuk nel Kurdistan iracheno, la cui perdita significherebbe per l'Iraq la rinuncia a buona parte delle risorse petrolifere e delle scarse risorse agricole di cui dispone ${ }^{8}$.

Ragioni «percettive». Ma la ragione più importante, e quella su cui convergono quelle che abbiamo già visto, è l'immagine che ogni parte si è fatta delle altre. «"Gentili ed ebrei - si legge in un romanzo israeliano - sono come uomini e donne", diceva mio padre. "Perché?" gli chiesi una volta. "Solo a causa di pregiudizi. Di tutte e due le parti, su sé e anche sull'altro" sorrise mio padre. "Ogni parte ha la sua immagine," diceva. "La sua immagine dell'altro. Così, quando ci si rivolge a qualcuno dell'altra parte, si parla all'immagine e non alla persona"» (Feldman 1987).

${ }^{8}$ Questo aspetto spiega la durissima battaglia per il possesso di Kirkuk, che è rimasta fuori della zona controllata dai curdi, e l'inflessibilità del governo iracheno sulle sorti del Sud-Ovest sciita, che è ancora più ricco di risorse petrolifere e per di più apre all'Iraq l'unico sbocco al mare. 
Quello che rende così persistenti queste immagini è il fatto che esse hanno un fondamento nella realtà. Non si tratta di semplici invenzioni o di steoreotipi confezionati dalla propaganda, ma del risultato di esperienze sociali, politiche o culturali che si sono «cristallizzate» e frappongono il loro schermo alle esperienze successive. L'immagine che un gruppo ha formato di sé o di un altro in circostanze particolari tende ad acquistare autonomia e a persistere, anche quando il mutamento delle circostanze consentirebbe di formare un'immagine diversa'.

L'elemento-base della rappresentazione delle minoranze è il legame che esse hanno o possono sempre avere col nemico: o perché lo stato ha vissuto qualcosa di simile nel passato, o perché lo sta sperimentando nel presente, o perché si limita a riportare ciò che ha appreso dall'esperienza di altri stati, le minoranze sono viste come il riflesso «interno» del nemico «esterno». In questo processo semplificatorio è l'immagine del nemico che forma l'immagine delle minoranze. La loro tragedia sta prima di tutto in questa impossibilità di essere viste per quello che sono all'interno dello stato - dei gruppi svantaggiati (politicamente, economicamente o socialmente) che chiedono il rispetto della loro diversità - senza essere confuse con quello che minaccia lo stato dall'esterno.

Anche da questo lato difficilmente la guerra del Golfo migliorerà la situazione. L'esperienza, traumatizzante per Israele, della minoranza araba che festeggia ogni lancio degli Scud iracheni su Tel Aviv, e quella non meno traumatizzante per gli arabi iracheni ma probabilmente, in futuro, anche per gli altri arabi, della minoranza curda che approfitta della sconfitta per rafforzare le sue posizioni e ottenere l'appoggio della coalizione nemica, sono destinate a pesare a lungo sull'immagine dei gruppi minoritari in Medio Oriente.

Un secondo elemento importante nella percezione delle minoranze, legato proprio alla loro diffusione, è l'estrema sensibilità di ogni stato all'evoluzione degli altri. In questo senso si può parlare di «interdipendenza», ma liberando il termine da ogni valenza ottimistica o pacifica: si tratta, piuttosto, di una interdipendenza della paura e del conflitto.

Il fatto che lo stesso problema sia affrontato da diversi stati con diverse minoranze fa sì che l'insicurezza di ciascuno venga

9 Come esempio rimarchevole di persistenza ma anche di evoluzione dell'immagine, cfr. l'immagine dei turchi nel mondo arabo in Haarmann (1988). 
alimentata dall'insicurezza degli altri (Weinbaum 1987). Il timore di «reazione a catena» è il secondo meccanismo semplificatorio ad entrare in gioco: non importa che tutte le minoranze siano in realtà diverse, perché tutto quello che accade ad una ha (o può avere) effetti sulle altre. L'immagine di ogni minoranza è anche, almeno in parte, il riflesso delle altre minoranze, nel senso che quello che ciascuna compie o ottiene in uno stato influenza in diversi gradi l'atteggiamento degli altri stati.

$\mathrm{Ma}$ quello che contribuisce più di tutto a complicare le cose è, paradossalmente, l'impossibilità di concordare su chi è maggioranza e chi è minoranza. La regola non è, infatti, la competizione di due gruppi in uno spazio definito ma piuttosto una serie concentrica di maggioranze e di minoranze nella quale ogni maggioranza è, nel cerchio più ampio, minoranza, mentre ogni minoranza è, nel cerchio più stretto, maggioranza. Il conflitto tra maggioranza e minoranza è prima di tutto un conflitto su chi «fissa l'orizzonte», poiché a ogni mutamento dell'orizzonte cambia la distribuzione delle parti.

La loro reciproca diffidenza è peggiorata dal fatto che ogni gruppo tende a collocare il conflitto nel cerchio in cui è minoranza e a non comprendere che l'altro gruppo può fare esattamente lo stesso. Mentre tutte e due le parti sono per qualcosa maggioranza e per qualcosa minoranza, ciascuna vede se stessa solo come minoranza e l'altra solo come maggioranza. L'immagine dell'assedio, che è l'unica cosa sulla quale concordano, non deve fare dimenticare che quello che sperimentano è un assedio paradossale, nel quale tutti i partecipanti si riconoscono come assediati ma non possono impedire che gli altri vedano in loro gli assedianti.

Questa «doppia immagine» (Heraclides 1989, 206) si è imposta, innanzitutto, nei due casi attorno a cui hanno ruotato alcuni tra i più persistenti conflitti della regione: quello dei cristiani in Libano, almeno fino a quando hanno conservato la supremazia politica, e quello degli ebrei in Israele. Il conflitto di prospettive non potrebbe essere più radicale. Da una parte gli ebrei (e i cristiani) si sentono minoranza perché tengono lo sguardo fisso sulla regione, nella quale costituiscono una piccola enclave non-araba (e non-islamica) in uno spazio dominato dagli arabi. Dall'altra parte gli arabi di Israele si percepiscono nello stesso modo o perché restringono l'orizzonte ai confini dello stato ebraico, entro il quale sono loro ad essere politicamente, socialmente e numericamente minoritari, o perché lo al- 
largano all'intero sistema internazionale, nel quale tornano a vedere in se stessi la parte assediata e in Israele la punta avanzata dell'assedio. Sia gli uni che gli altri hanno le loro ragioni per dare al conflitto l'orizzonte che gli danno, se non che facendolo non riconoscono che l'altra parte gli dà un orizzonte diverso.

L'ultimo elemento, strettamente legato a questo, è quella che si può definire una «piramide della paura». A mano a mano che lo stato li combatte, i gruppi minoritari tendono a comportarsi al loro interno nello stesso modo in cui lo stato si comporta con loro. «Ogni volta che A opprime B» scriveva George Orwell «è chiaro alla gente di buon senso che $\mathrm{B}$ dovrebbe essere indipendente, se non che c'è un altro gruppo, $\mathrm{C}$, che è ansioso di essere indipendente da B» (cit. in Ryan 1988, 173). Il momento in cui si afferma la propria identità è il momento meno adatto a riconoscere altre identità al proprio interno. In una situazione come quella mediorientale in cui maggioranze e minoranze sono così saldamente intrecciate, questo significa che l'intolleranza di ognuno può trovare continue conferme nell'intolleranza dell'altro e rappresentarsi in tranquilla coscienza come un atteggiamento difensivo.

\section{Conclusioni}

Questa confusione tra condizione delle minoranze e preoccupazioni di «sicurezza» richiama l'incessante rapporto che esiste tra politica interna e politica internazionale. Per capire che cosa rende così difficile la convivenza è necessario tenere a mente che ogni gruppo minoritario si trova nel mezzo di un gioco a più dimensioni, delle quali una è costituita dall'interazione con gli altri gruppi dell'arena politica interna, e le altre dai diversi elementi della competizione regionale.

Il principale vantaggio di questa impostazione è quello di abbattere il «recinto metodologico» nel quale è spesso chiusa l'analisi delle vicende mediorientali, che preferisce ancora far leva su elementi «riduzionistici» come l'autoritarismo dei paesi della regione, la personalità dei leader o le caratteristiche della cultura araba e/o islamica, piuttosto che adottare il tipo di spiegazione che si adotterebbe in altri contesti per spiegare le stesse cose.

L'altro lato della stessa medaglia è che, una volta operato questo capovolgimento, lo studio delle minoranze in Medio 
Oriente offre indicazioni utili a quello degli altri sistemi regionali caratterizzati, come quello mediorientale, da una elevata instabilità e dalla compresenza di fattori unitari e di divisione. In questo ambito rientrano, per esempio, i sistemi politici internazionali che stanno sorgendo o sono già sorti sulle ceneri di sistemi politici interni di carattere imperiale (come l'impero sovietico) o federale (come la Jugoslavia), e nei quali la divisione dei beni che appartenevano al complesso venuto meno, la trasformazione della natura dei confini e la presenza di ampie enclave minoritarie stanno creando problemi di sicurezza e di percezione per certi versi simili a quelli che abbiamo osservato.

La confluenza di politica interna e politica internazionale ha diverse gradazioni, lungo un continuum che procede dalla più debole alla più forte.

Il significato minimo è facile da accettare: non è possibile comprendere la situazione dei gruppi minoritari finché la si studia come un problema esclusivamente interno. Fino a questo punto si tratta solo di constatare l'interdipendenza e la reciproca permeabilità tra politica interna e politica internazionale. La continua interazione tra interno ed esterno comporta che nessuno stato possa prescindere dagli effetti che l'esistenza di una minoranza può avere sulla competizione con gli altri stati.

Contiguo a questo primo significato ce n'è un altro, già più impegnativo, con il quale si entra nell'ambito delle teorie sistemiche: la politica repressiva nei confronti delle minoranze non è esercitata solo a causa della mentalità orientata al potere degli stati autoritari ma anche a causa degli imperativi politico-internazionali. Come ogni teoria sistemica, questa tesi spiega perché regimi diversi tendano a comportarsi nello stesso modo in situazioni simili, mentre regimi simili tendono a comportarsi diversamente in situazioni diverse. Grazie ad essa si può spiegare e prevedere la continuità all'interno del sistema (Waltz 1979): poiché il modo come lo stato risponde alle rivendicazioni delle minoranze non dipende solo dalla sua struttura interna ma anche dalle caratteristiche del sistema regionale, cambiare il regime politico di uno stato non è sufficiente a migliorare la condizione delle minoranze.

Il terzo significato procede da questo punto ma opera una rottura: fino a quando il sistema mediorientale avrà le caratteristiche che ha avuto fino ad ora, l'esistenza delle minoranze sarà vista alla luce delle preoccupazioni di sicurezza. Il progresso rispetto all'accezione di prima non è di poco conto: le caratteri- 
stiche del sistema regionale non si limitano più a condizionare la politica degli attori, ma esercitano una sorta di primato sulle loro caratteristiche interne. In una situazione dominata dalle difficoltà dei rapporti di ciascuno stato con gli altri le richieste delle minoranze cadono vittima del gioco che impone agli stati di premunirsi contro tutto quello che potrebbe indebolirli. La prevalenza delle preoccupazioni di sicurezza non esclude ancora che stati diversi (per esempio: stati democratici o autoritari) possano adottare politiche diverse, ma spiega perché i gruppi minoritari non riescano a farsi riconoscere per quello che sono all'interno dello stato senza essere confusi con quello che minaccia lo stato dall'esterno.

Il quarto significato, il più estremo, sfocia in quello che è stato definito «determinismo situazionale» (Latsis 1976). Il peso rispettivo di politica interna e politica internazionale non è sempre lo stesso (Bonanate 1986). Il loro rapporto è regolato da una sorta di «cerniera» (il sistema) che lo fa funzionare come le due lame di una forbice: in condizioni «normali» le uniche influenze esterne sullo stato sono quelle «naturali» delle interazioni economiche e commerciali, del turismo e così via. Ma quanto più cresce la rilevanza internazionale di un evento tanto più la forbice si chiude e la politica internazionale prevale su quella interna: questo avviene non solo nel caso dello scoppio di una guerra, ma anche in quelle situazioni intermedie tra la pace e la guerra (Lebow 1981) che sono le «crisi internazionali». In queste circostanze, quando lo stato percepisce una minaccia ai propri valori fondamentali (Hermann 1972), le caratteristiche interne smettono del tutto di contare perché è la situazione che impone di subordinare ogni altra considerazione agli imperativi di sopravvivenza. In presenza di una costrizione estrema, come nel caso dello scoppio di un incendio in una casa con un'unica uscita o di una insurrezione interna appoggiata dall'esterno, «l'analisi del processo decisionale sarebbe utile solo se riferita ad individui che decidessero di rimanere dove sono» (Wolfers 1962) o a stati che scegliessero di non difendersi.

Il rigetto delle interpretazioni riduttivistiche non cancella l'importanza dell'adesione a certi valori invece che ad altri, ma segnala il limite oltre il quale essa non si può spingere. Quando si valuta il comportamento degli attori in base alla maggiore o minore conformità a un principio generale non si tiene conto che nella realtà gli attori non si trovano tutti nella stessa condizione, e che in condizioni diverse aderire al principio non vuole 
dire sempre la stessa cosa (e non espone sempre alle stesse perdite).

Malgrado le aspettative suscitate dalle speranze di democratizzazione e dall'adozione del principio d'intervento a fini umanitari nel Kurdistan iracheno, la condizione delle minoranze in Medio Oriente resta ancora prigioniera di questo «dilemma della sicurezza». Per certi aspetti, anzi, la fine dell'ordine bipolare e la guerra contro l'Iraq hanno persino rafforzato l'insicurezza degli stati della regione, confermando la dimensione internazionale del problema delle minoranze e mettendo a nudo la relazione che esiste tra i due processi.

La ripresa della competizione è stata innescata dal collasso dell'Iraq, dall'irruzione di nuovi attori (dai curdi agli stati asiatici dell'ex Unione Sovietica) e di nuove poste in gioco (le risorse idriche), e dall'aggravamento della storica contraddizione tra la prevalenza numerica degli attori (stati e popoli) arabi e la prevalenza politica, militare ed economica dei tre attori non-arabi: Turchia, Israele ed Iran; questa ripresa della competizione si è così accompagnata ad una nuova e più radicale crisi dei confini. Il collasso dell'«ordine» bipolare tende a riannodarsi a quello dell'ordine ottomano e di quelli coloniali, rimettendo in discussione le sistemazioni quasi sempre irrealistiche che li hanno seguiti e rivelando retrospettivamente che il principio dell'assoluta intangibilità dei confini adottato dalle organizzazioni regionali poteva stare in piedi solo perché rientrava anch'esso nella «pace armata» delle superpotenze.

L'effetto più appariscente di questa crisi è quello di creare due nuove forme di instabilità. Da un lato essa toglie «serietà» ai confini esterni e incoraggia gli stati più forti (come l'Iraq e la Siria) ad assorbire quelli più deboli (come il Kuwait e il Libano). Dall'altro, e per lo stesso motivo, essa espone tutti gli stati della regione a forti spinte centrifughe, come quelle che stanno mettendo in forse la sopravvivenza dell'Iraq come stato unitario.

Questo processo rimette tutti gli stati della regione di fronte a quella che abbiamo indicato come la causa fondamentale della loro insicurezza: il carattere artificiale dei confini e, pertanto, della loro identità. Le convulsioni della regione mesopotamica con l'annessione irachena del Kuwait prima e poi, come contraccolpo, con l'inedito «regime» impiantato nel Kurdistan iracheno - il quale non è ancora uno stato ma sottrae all'Iraq una parte delle prerogative normali della sovranità - hanno trascina- 
to con sé il riacutizzarsi del separatismo curdo in Turchia, l'indebolimento strisciante del principio dell'inviolabilità dei confini per le continue incursioni turche in Iran e in Iraq a caccia dei guerriglieri curdi, la riapertura di innumerevoli contenziosi territoriali tra Arabia Saudita e Yemen, Arabia Saudita e Qatar, Iran e Emirati Arabi Uniti, Iran e Bahrein ${ }^{10}$.

Come se non bastasse, la guerra del Golfo ha riportato in superficie una dimensione spesso trascurata, anche se politicamente fondamentale, di questa artificialità. La facilità con la quale le truppe irachene hanno occupato il Kuwait, l'incapacità degli altri paesi della regione di opporre una reazione e l'intervento risolutore della coalizione guidata dagli Stati Uniti hanno dimostrato ancora una volta che solo un (continuo) intervento esterno può stabilizzare la carta politica del Medio Oriente mentre questo, se da una parte riesce a sanzionare «l'inviolabilità» delle divisioni, dall'altra ne rinnova continuamente il carattere artificiale, esogeno e nel linguaggio polemico, ancora una volta, «coloniale». L'identità debole dei paesi mediorientali non esce da questo paradosso: da una parte, essa sconta il vizio d'origine di confini ereditati dal dominio straniero (ottomano e coloniale), dall'altra parte non può fare a meno di rinnovare questo vizio se vuole tenere in piedi i confini. L'insicurezza dei paesi della regione non deriva solo dal fatto che non esistono ragioni storiche, culturali o linguistiche perché i confini cadano là dove cadono e non altrove, ma anche dal fatto che ogni volta che qualcuno decide di spostarli in avanti (come Saddam Hussein) o all'indietro (come i curdi) nessuno di loro è in grado di impedirlo.

L'impossibilità di contare su divisioni certe rende ancora più evidente la reciproca permeabilità tra politica interna e politica internazionale. Il risveglio delle minoranze interagisce in diversi modi con la crisi dei confini.

La modalità più superficiale è quella solita della strumentalizzazione del problema a vantaggio del nemico. Da un estremo all'altro della regione, l'esperienza degli ultimi mesi confer-

${ }^{10}$ Questa precarietà è stata riassunta in termini brutali dal Ministero degli Esteri iraniano il quale, rispondendo alle proteste della Lega Araba per l'annessione dell'isola di Abu Musa, ha ammonito che le rivendicazioni arabe sulle isole «potrebbero aprire il vaso di Pandora delle rivendicazioni territoriali nella regione», e che se si decidesse di risalire a confini storicamente meno arbitrari degli attuali «la sovranità dell'Iran si estenderebbe su ampie parti dell'Iraq e sull'intero Bahrein» («Frankfuerter Allgemeine», 30 settembre 1992). 
ma sia la tendenza all'internazionalizzazione dei conflitti interni sia il ruolo che le minoranze possono giocare nell'ingerenza straniera. Non importa che in alcuni casi esse vengano utilizzate solo come strumento di pressione (come nel sostegno dei paesi arabi moderati ai gruppi autonomisti in Sudan, o nell'appoggio siriano ai guerriglieri curdi in Turchia in risposta ai progetti turchi di sfruttamento dell'Eufrate), mentre in altri diventino un mezzo per dividere o minacciare di dividere lo stato (come nella politica degli Stati Uniti in Iraq). Quello che conta è che in un caso e nell'altro l'esistenza delle minoranze prefigura una sorta di «apertura» nel confine, un varco nel quale lo stato si sente più debole di fronte ai suoi (possibili) nemici.

Ma la dimensione più importante è rivolta, invece che all'esterno, all'interno dello stato. Lo scoppio della guerra civile in Iraq all'indomani della guerra del Golfo ha rivelato in termini drammatici che l'esistenza delle minoranze disegna confini virtuali, trasversali rispetto a quelli dello stato, e destinati a riemergere ogni volta che il confine dello stato si indebolisce. Il problema delle minoranze diventa tutt'uno con il problema dei confini. In un senso, perché mette allo scoperto le stratificazioni di cui sono composti gli stati mediorientali e il fatto che esse avrebbero potuto (e potrebbero ancora) essere combinate in modo diverso. Nell'altro senso, soprattutto, perché le enclave minoritarie vengono a costituire una sorta di «zona grigia» tra politica interna e politica internazionale, un luogo che non è più del tutto all'interno dello stato ma non ne è ancora del tutto al di fuori, e dove si combatte (come nel territorio che ha materializzato questa ambiguità, il Kurdistan iracheno) per decidere a quale delle due sfere si finirà per appartenere.

\section{Riferimenti bibliografici}

Allison, G. (1971), Essence of Decision. Explaining the Cuban Missile Crisis, Boston, Little, Brown \& Co.

Balladore Pallieri, G. (1964), Dottrina dello Stato, Padova, Cedam.

Baram, A. (1990), Territorial Nationalism in the Middle East, in «Middle Eastern Studies», XXVI, pp. 425-448.

Bonanate, L. (1986), Politica internazionale e politica interna: reciproche limitazioni, in L. Bonanate e C.M. Santoro (a cura di), Teoria $e$ analisi nelle relazioni internazionali, Bologna, Il Mulino, pp. 85105. 
Brinner, W.B. (1987), The Arabs of Israel: The Past Twenty Years, in «Middle East Review», XX, pp. 13-21.

Brown, D. (1989), Ethnic Revival: perspectives on state and society, in «Third World Quarterly», XI, pp. 1-17.

Cachia, P. (1989), The "Other» in Modern Arabic Prose Writing, in «Middle East Review», XXII, pp. 41-46.

Cahen, C. (1969), L'Islamismo (I). Dalle origini all'inizio dell'Impero ottomano, in AA.VV., Storia universale Feltrinelli, vol. XIV.

Cataluccio, F. (1977), La questione d'Oriente: lotte di nazionalità e interessi di potenze (1815-1965), in AA.VV., Nuove questioni di storia contemporanea, II, Marzorati, pp. 1467-1533.

Clay, J.W. (1989), Epilogue: the ethnic future of nations, in «Third World Quarterly», XI, pp. 223-233.

Cohen, S. (1986), The Geopolitics of Israel's Border Question, Tel Aviv, JCSS Studies, Tel Aviv University.

Cremonesi, L. (1985), Le origini del sionismo e la nascita del kibbutz (1881-1920), Firenze, Giuntina.

Entessar, N. (1989), The Kurdish Mosaic of Discord, in «Third World Quarterly», XI, pp. 83-100.

Feldman, Y.S. (1989), The «Other Within» in Contemporary Israeli Fiction, in «Middle East Review», XXII, pp. 47-53.

Francis, E.K. (1976), Interethnic Relations, New York, Elsevier.

Freedman, L. (1991), The Gulf War and the New World Order, in «Survival», XXXIII, pp. 195-209.

Grunebaum, G.E. Von (1972), L'Islamismo (II). Dalla caduta di Costantinopoli ai nostri giorni, in AA.VV., Storia universale Feltrinelli, vol. XV, Feltrinelli, Milano.

Hagopian, E.C. (1989), From Maronite Hegemony to Maronite Militancy: the Creation and Disintegration of Lebanon, in "Third World Quarterly», XI, pp. 101-118.

Haarmann, U.W. (1988), Ideology and History, Identity and Alterity: The Arab Image of the Turk from the Abbasid to Modern Egypt, in «International Journal of Middle Eastern Studies», XX, pp. 175 . 196.

Heraclides, A. (1989), Conflict Resolution, Ethnonationalism and the Middle East Impasse, in «Journal of Peace Research», XXVI, pp. 197-212.

Hermann, C.F. (a cura di) (1972), International Crisis: Insights from Bebavioral Research, New York, The Free Press.

Herz, J. (1951), Political Realism and Political Idealism, Chicago, Chicago University Press.

Hottinger, A. (1988), Der «Aufstand» der Araber in den besetzen Gebieten, in «Europa Archiv», XI, pp. 299-310.

Hourani, A. (1962), Arabic Thought in the Liberal Age 1798-1939, London, Oxford University Press. 
Jervis, R. (1978), Cooperation under the Security Dilemma, in «World Politics», XXX, pp. 167-214.

Klein, M. (1990), Arab Unity: A Nonexistent Entity, in «The Jerusalem Journal of International Relations», XII, pp. 28-44.

Latsis, S.J. (1976), A Research Programme in Economics, in S.J. Latsis (a cura di), Method and Appraisal in Economics, Cambridge, Cambridge University Press, pp. 1-41.

Lebow, R.N. (1981), Between Peace and War: The Nature of International Crisis, Baltimore, The Johns Hopkins University Press.

Moragh, G. (1989), The Arab as «Other» in Israeli Fiction, in «Middle East Review», XXII, pp. 35-40.

Moutafakis, G. (1986), The Role of Minorities in the Modern Middle East Societies, in M. Curtis (a cura di), The Middle East Reader, New Brunswich, Transaction Books.

NES (1990-1991), Minoranze in Medio Oriente, n. 23/24.

Peeters, Y.J.D. (1987), The Rights of Minorities in Present-day Turkey, in «Europa Ethnica», III, pp. 131-138.

Riggs, F.W. (1988), Modes of Ethnicity, in «Europa Ethnica», IV, pp. 161-177.

Rodinson, M. (1980), Gli Arabi. Storia, caratteri, ideologia, prospettive, Firenze, Sansoni.

Ryan, S. (1988), Explaining ethnic conflict: the neglected international dimension, in «Review of International Studies», XIV, pp. 161177.

Simmel, G. (1955), Conflict, New York, The Free Press.

Singer, D.J. (1969), The Level of Analysis Problem in International Relations, in J.N. Rosenau (a cura di), International Politics and Foreign Policy, New York, Free Press, pp. 20-29.

Smith, A.D. (1981), The Ethnic Revival, Cambridge, Cambridge University Press; trad. it. Il revival etnico, Bologna, Il Mulino.

Snyder, G.H. (1984), The Security Dilemma in Alliance Politics, in «World Politics», XXXVI, pp. 461-495; trad. it. Il dilemma della sicurezza nella politica delle alleanze, in L. Bonanate e C.M. Santoro (a cura di), Teoria e analisi nelle relazioni internazionali, Bologna, Il Mulino, 1986, pp. 351-389.

Tocqueville, A. de (1981), L'Antico Regime e la rivoluzione, Milano, Rizzoli.

Waltz, K.N. (1979), Theory of International Politics, Reading, Addison-Wesley; trad. it. Teoria della politica internazionale, Bologna, Il Mulino, 1987.

Weinbaum, M.G. (1987), The Internationalization of Domestic Conflict in the Middle East, in «Middle East Review», XX, pp. 31-42.

Wolfers, A. (1962), Discord and Collaboration. Essays on International Politics, Baltimore, The Johns Hopkins University Press. 